\title{
On the development of miransertib for rare diseases: an interview with ArQule's Brian Schwartz
}

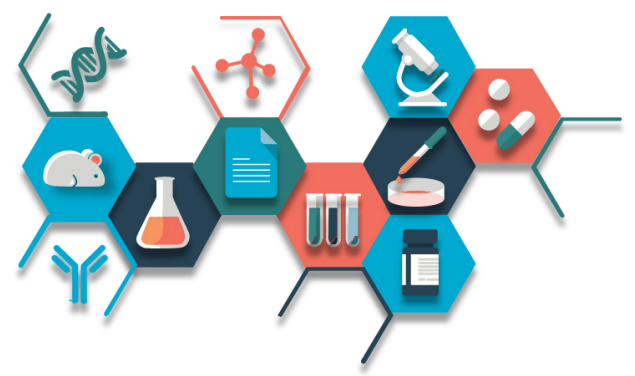

\author{
Brian Schwartz ${ }^{1}$ \\ ${ }^{1}$ ArQule Inc. Burlington, MA 01803-4769, USA
}

Brian Schwartz is CMO of ArQule, a clinical-stage biopharmaceutical company developing small-molecule kinase inhibitors for precision medicine oncology and rare diseases. In this interview he tells us more about the company's current projects, which include drug development for the rare diseases Proteus syndrome and PROS.

First draft submitted: 16 December 2019; Accepted for publication: 16 December 2019; Published online: 10 February 2020

Keywords: AKT inhibitors • drug development $\bullet$ drug discovery $\bullet$ Miransertib • overgrowth diseases $\bullet$ PIK3CArelated overgrowth spectrum • Proteus syndrome $\bullet$ rare diseases

\section{Would you be able to tell us about ArQule, what its mission is \& what is special about it?}

ArQule (MA, USA) is a company that specializes in discovering and developing drugs, using personalized medicine as our goal. We look for drugs that target specific genetic events and we have been able to bring a number of them into the clinic in rare diseases and oncology.

Approximately 25 years ago, ArQule was formed as a combinatorial chemistry company, which would make molecules for companies like Pfizer and Bayer. We have a library that is about a couple of million compounds strong, which we identified for Big pharma who would then use the library as a high-throughput screen.

As these companies moved their technology to other countries, ArQule realized it would have to change. So, ArQule changed from a chemistry company to a development company, which is really focused on kinase inhibitors.

One project that we are interested in at the moment is protein kinase B (AKT) inhibitors that target rare diseases with both $A K T$ mutations, as well as upstream PI3K mutations, which cause the rare overgrowth diseases of Proteus syndrome and PIK3CA-related overgrowth spectrum (PROS). We also have a Bruton's tyrosine kinase (BTK) inhibitor, which not only works against $B T K$ wild-type but also against a mutation, C481, which causes treatment resistance in patients who have received first-generation BTK inhibitors for diseases such as chronic lymphocytic leukemia or B-cell lymphomas. We also have an FGFR inhibitor, which is currently being evaluated in patients with genetic aberrations of FGFR2 and FGFR3 in cholangiocarcinoma and bladder cancer.

\section{What is Proteus syndrome \& PROS?}

Proteus syndrome and PROS are rare mosaic disorders. Early on in utero, a small islet of cells acquires an unusual mutation, such as $A K T 1$ or $P I 3 K$, which mature as the embryo grows. After birth, these children often appear normal; however, as they continue to develop these little islets grow at a much higher rate than the rest of the body. These islets can be found throughout the body, causing a variety of case presentations. For example, you can have a little islet of ten cells or you can have a whole big wad of cells. Therefore, some patients can present with a large finger, or one large foot, or a large lipoma on their back, whereas other people are completely disformed. The worst case is the famous Elephant Man disease where they have significant overgrowth in almost all different parts of their body. They are very varied and heterogeneous diseases.

We have taken our inhibitor, which is a potent selective inhibitor of AKT, and tested it preclinically in cells from these patients and discovered that even at very low concentrations it inhibits the growth. We are now in the last phase of testing within the clinic to see if it is going to work in a broader variety of patients. 


\section{You recently presented new data on a new drug called Miransertib for Proteus syndrome \& PROS, would you be able to explain this data to our audience?}

One of the areas that is affected by this overgrowth is the vasculature of the vessels due to either $P I 3 K$ or $A K T$ mutations. We then engineered the most common PI3K mutation into the vasculature and treated one group of animals. At the same time, we engineered the mutation within a second group of animals and waited for it to become established and then treat it.

In both settings, we were able to show that Miransertib either prevented the vessels from being formed or caused regression of the vessels once they had been formed. This is very important as it suggests that we can stop the disease in its tracks, or at least there is the hope of shrinking the disease and suggests that in the clinic we will be able to decrease the size, or change the vasculature so that there would be an opportunity to improve the disease. That was the poster we presented at ASHG 2019 [1]; it is preclinical, but it is quite a predictive model for both AKT, PI3K and mTOR inhibitors.

\section{What are the current options that are available for these patients \& how much could Miransertib potentially do?}

Proteus is caused by a rare $A K T$ mutation that is much rarer than the PI3K mutation. Approximately $25 \%$ of patients with $A K T$ mutations will die before the age of 20, from either clotting or vascular abnormalities, or due to the fact that they have a high risk of developing certain types of cancers.

Initially, we performed a 'Phase 0' study at the NIH. As there were no animal models for Proteus disease, we administered the lowest possible dose of the drug into six patients. We removed tissue before and after drug administration to determine if the signal in these affected areas decreased and if we could determine end points. We found that the signal did decrease and surprisingly, in the younger patients who were growing, we were able to reverse some of the growth that had already been established.

From these data we were able to start a clinical trial (NCT03094832) where the US FDA agreed that if we could show a decrease of certain lesions in Proteus patients, plus an improvement in quality of life, the drug would be approved. This study is still ongoing.

The second group of patients is the PROS patients. Here we are not dealing with one mutation like Proteus, we are dealing with multiple PI3K mutations and therefore many types of clinical presentations. Initially we enrolled 17 patients and we were able to dose escalate and determine a safe and efficacious dose. Now we have expanded that trial (NCT03094832) as we saw patient improvement and better outcomes. Although we did not see as dramatic shrinkage as we saw with the Proteus group, we did notice that the lesions were changing. Patients who had lesions growing aggressively saw their lesions stay the same size while on the drug. In addition, we did see patients with significant improvements in symptoms and improvements to their quality of life. For example, we had a patient with intra-abdominal lesions who had to be fed via a G-tube as the lesions increased the pressure on his stomach. After a couple of months, the patient could eat without the G-tube.

This study is expanding and we have just opened sites in Australia, Spain and Italy. Hopefully we will be to get an answer for these patient's questions: can we halt their disease progression? Can we shrink their disease? And can we get this drug approved in the next couple of years? We will be evaluating these lesions using MRIs for Proteus syndrome, performing volumetric MRIs and looking at the change in those MRIs overtime for the PROS disease.

When we started in oncology, we quickly realized that these rare groups of somatic disorders are a really important and interesting group of rare diseases to study with kinases. As new mutations are being discovered, for example, $R A S$ and $R A F$, it opens up to doors for further therapeutic discovery and development for these rare overgrowth and vascular disorders.

\section{Disclaimer}

The opinions expressed in this interview are those of the interviewee and do not necessarily reflect the views of Future Medicine Ltd.

Financial \& competing interests disclosure

B Schwartz is Chief Medical Officer of ArQule, which is funding and advancing the research discussed in the interview. The author has no other relevant affiliations or financial involvement with any organization or entity with a financial interest in or financial conflict with the subject matter or materials discussed in the manuscript apart from those disclosed.

No writing assistance was utilized in the production of this manuscript. 


\section{Open access}

This work is licensed under the Attribution-NonCommercial-NoDerivatives 4.0 Unported License. To view a copy of this license, visit http://creativecommons.org/licenses/by-nc-nd/4.0/

\section{Reference}

1. Kobiatka P, Sabata H, Yu Y, Schwartz B, Graupera M, Castillo SD. Miransertib (ARQ 092) prevents the formation of PIK3CAH1047R-driven vascular malformations in mice. Presented at: American Society of Human Genetics Annual Meeting. TX, USA, 15-19 October 2019. 